\title{
Hadron Polarization in Semi-Inclusive Reactions Involving Photons
}

\author{
C.J. Solano Salinas and V. Gupta \\ Depto. Física Aplicada, CINVESTAV del I.P.N. Unidad Mérida, México
}

Received on 7 October, 2003

\begin{abstract}
A phenomenological model which has had some success in explaining polarization phenomena and left-right asymmetry in inclusive proton-proton scattering is considered for reactions involving photons. In particular, the reactions (a) $\gamma+p \rightarrow H+X$; (b) $\gamma+p(\uparrow) \rightarrow \pi^{ \pm}+X$ and (c) $p(\uparrow)+p \rightarrow \gamma+X$ are considered where $\gamma=$ resolved photon and hyperon $H=\Lambda, \Sigma, \Xi$ etc. Predictions for hyperon polarization in (a) and the asymmetry (in (b) and (c)) provide further tests of this particular model. Feasibility of observing (b) at HERA and the effect of the polarization of the sea in the proton in $p(\uparrow)+p \rightarrow \pi^{ \pm}+X$ is briefly discussed.
\end{abstract}

\section{Introduction}

Polarization phenomena in high energy proton-proton scattering has been studied experimentally [1] and theoretically [2,3] for over two decades. Left-right asymmetry $\left(A_{N}\right)$ has been measured [4] in the scattering of a transversely polarized proton $(p(\uparrow))$ with an unpolarized proton $(\mathrm{p})$ in the semi inclusive reactions of the type $p(\uparrow)+$ $p \rightarrow h+X$, where $h$ is a hadron and typically is $\pi^{ \pm}, \Lambda$ etc. Significant transverse polarization $P(H)$ for hyperons $H=\Lambda, \Sigma, \Xi$ is found in unpolarized proton-proton inclusive scattering, viz, $p+p \rightarrow H+X$. The effects observed in the above reactions are non-perturbative by nature and hence have not been accessible to calculations based on first principles, that is, quantum chromodynamics. Phenomenological models have been suggested for the production of observed hadron.

Of the various models, which reproduce some features of the data for $A_{N}$ and $P(H)$, the most successful one is perhaps the "orbiting valence quark model" [3] and is very interesting in that it relates $A_{N}$ and $P(H)$ as due to the same underlying production mechanism in terms of the constituent quarks of the protons and the produced hadron.

The basic idea is that a transversely polarized quark $q^{P}(\uparrow)$ or $q^{P}(\downarrow)$ in the projectile (P) proton combines with the appropriate quarks or anti-quarks from the 'sea' of the target proton to form the observed hadron $h$ in the reaction Eq (1). Further, the produced hadron $h$ moves preferentially to the left of the beam direction, in the upper side of the production plane. That is if $\vec{S} \cdot \vec{n}>0$, where $\vec{S}$ represents the transverse polarization of $q^{P}(\uparrow)$, which is polarized upward of the production plane. While, $h$ formed from $q^{P}(\downarrow)$, with $\vec{S} \cdot \vec{n}<0$, will move preferentially to the right. This gives rise to a left-right asymmetry. The same reasoning gives rise to a net $P(H)$ in the sub-sample of hyperons going left since $\mathrm{H}$ is assumed to retain the polarization of the $q^{P}$ which forms it [3]. Based on these ideas, we consider other reactions to provide further tests of this model.

\section{Resolved photon-proton reactions}

We consider the transverse polarization of the hyperon $H$ in $e^{-}+p \rightarrow e^{-}+H+X$. Here the effective reaction is $\gamma+p \longrightarrow H+X$, where the $p$ is unpolarized and $H=\Lambda, \Sigma^{ \pm}$, etc. The ee $\gamma$ vertex is considered to be 'real' with 'resolved' hadronic components.

The kinematics is similar to that for reaction in $\gamma+p(\uparrow) \rightarrow h+X$, except that here the hyperon polarization $\mathrm{P}(\mathrm{H})$ is measured. To see how $\mathrm{P}(\mathrm{H})$ arises in the model, take the direction of the proton beam in cm-frame. Let transverse polarization of a quark (in the proton) be denoted by $\uparrow$ or $\downarrow$, perpendicular to the production plane. One assumes [3], that a quark with upward ( $\uparrow$ ) (downward $(\downarrow))$ polarization will preferentially scatter to the left (right) in the production plane, with respect to the beam direction.

This quark will combine with a two quark state $(q q)_{\gamma}$ from the photon to form the hyperon $\mathrm{H}$. It is also possible that two quarks from the proton combine with a quark from the photon to give the polarized $\mathrm{H}$. To clarify how the model works let us consider the production of $\Sigma^{-}$whose quark content is $d d s$. In this case, only the valence d-quark from the proton $\left(d_{v}^{p}\right)$ is common with those in $\Sigma^{-}$. Let the probability of $d_{v}^{p}(\uparrow)\left(d_{v}^{p}(\downarrow)\right)$ from the proton to move to the left (right) be $\alpha$. Then, the probability of $d_{v}^{p}(\uparrow)\left(d_{v}^{p}(\downarrow)\right)$ to move to the right (left) will be $(1-\alpha)$. The unpolarized proton has equal probability of having a $d_{v}^{p}(\uparrow)$ or $d_{v}^{p}(\downarrow)$. Using $S U(6)$ baryon wave functions, we know that the probability of $d \uparrow(d \downarrow)$ in a $\Sigma^{-}(\uparrow)$ is $\frac{5}{6}\left(\frac{1}{6}\right)$. We expect that $N\left(\Sigma^{-}(\uparrow)\right)$, the number of $\Sigma^{-}(\uparrow)$ formed by the left moving $\mathrm{d} \uparrow$ and $d \downarrow$, will be proportional to $\frac{5}{6} \alpha+\frac{1}{6}(1-\alpha)$, while the number $N\left(\Sigma^{-}(\downarrow)\right)$ will be proportional to $\frac{1}{6} \alpha+\frac{5}{6}(1-\alpha)$.

One expects the polarization $P\left(\Sigma^{-}\right)=$ $\frac{N\left(\Sigma^{-}(\uparrow)\right)-N\left(\Sigma^{-}(\downarrow)\right)}{N\left(\Sigma^{-}(\uparrow)\right)+N\left(\Sigma^{-}(\downarrow)\right)}=\frac{2}{3}(2 \alpha-1)$. As expected, this is zero if $\alpha=\frac{1}{2}$. Since, $\alpha$ is assumed to be $\geq \frac{1}{2}$, the model predicts $0 \leq P\left(\Sigma^{-}\right) \leq \frac{2}{3}$. Using $S U(6)$ wave functions, we analyze the expected polarization for other hyperons. 
(i) For $\Xi^{0}(u s s)$ and $\Xi^{-}(d s s)$, the $u_{v}^{p}$ and $d_{v}^{p}$ will contribute to their production by combining with the $(s s)_{\gamma}-$ state from the photon. Here the probability of $u \uparrow(u \downarrow)$ and $d \uparrow(d \downarrow)$, respectively, is $\frac{1}{3}\left(\frac{2}{3}\right)$. Then $N\left(\Xi^{0 \uparrow}\right)$ or $N\left(\Xi^{-\uparrow}\right)$ is $\propto\left[\frac{1}{3} \alpha+\frac{2}{3}(1-\alpha)\right]$, while $N\left(\Xi^{0 \downarrow}\right)$ or $N\left(\Xi^{-\downarrow}\right)$ is $\propto\left[\frac{2}{3} \alpha+\frac{1}{3}(1-\alpha)\right]$, so that $P\left(\Xi^{0}\right)=P\left(\Xi^{-}\right)=\frac{1}{3}(1-2 \alpha)$. The polarization is expected to be opposite in sign to $P\left(\Sigma^{-}\right)$but smaller by factor of two.

(ii) For $\Sigma^{+}(u u s)$ there are two formation mechanisms: (1) $u_{v}^{p}+(u s)_{\gamma}$ and $(2)(u u)_{v}^{p}+(s)_{\gamma}$. The first mechanism (as in the $\Sigma^{-}$case) will contribute $\frac{2}{3}(2 \alpha-1)$ to the polarization. If $\alpha_{2}$ is the probability the spin up diquark (uu) to move to the left then the second mechanism will contribute $\frac{2}{3}\left(2 \alpha_{2}-1\right)$. If $\mathcal{P}_{1}$ and $\mathcal{P}_{2}$ are the probabilities for $\Sigma^{+}$to be formed by the mechanisms (1) and (2) respectively then, one expects to have (8) $P\left(\Sigma^{+}\right)=\frac{2}{3}(2 \alpha-1) \mathcal{P}_{1}+\frac{2}{3}\left(2 \alpha_{2}-1\right) \mathcal{P}_{2}$.

There is no definitive prediction for the polarization without having a way to estimate $\mathcal{P}_{1}, \mathcal{P}_{2}$, and $\alpha_{2}$. However we expect that for high $x_{F}(\geq 0.8)$ the second mechanism will dominate [5] and that for medium values of $x_{F}(0.4-0.6)$ the first mechanism will dominate. Then, measuring experimentally the polarizations of $P\left(\Sigma^{-}\right)$and $P\left(\Sigma^{+}\right)$for this medium $x_{F}$ region it can be determined, approximately, the $\mathcal{P}_{1}$ (and so the $\mathcal{P}_{2}$ ) probability. Further, measuring the $P\left(\Sigma^{+}\right)$for high $x_{F}$ we can determine also $\alpha_{2}$.

\section{Conclusions}

In this paper, tests of a particular phenomenological model are given for a new process, in particular, relations like $0<P\left(\Sigma^{-}\right)<\frac{2}{3}, P\left(\Sigma^{-}\right)=-2 P\left(\Xi^{0}\right)$, and $P\left(\Xi^{0}\right)=$ $P\left(\Xi^{-}\right)=\frac{1}{3}(1-2 \alpha)$ provide new and simple tests of the model. The processes which provide these tests would probe small $\mathrm{x}$-region of the proton.

\section{Acknowledgment}

This work was done at the CINVESTAV Unidad Mérida and is supported by CONACYT of Mexico.

\section{References}

[1] A. Lesnik et al. Phys. Rev. Lett. 35, 770 (1975); G. Bunce et al. Phys. Rev. Lett. 36, 1113 (1976); K. Heller et al. Phys. Lett. 68B, 480 (1977); C. Wilkinson et al. Phys. Rev. Lett. 46, 803, (1981); L. Deck et al. Phys. Rev. D 28, 1 (1983); E.C. Dukes et al. Phys. Lett. B 193, 135 (1987); J. Duryea et al. Phys. Rev. Lett. 67, 1193 (1991); P.M. Ho et al. Phys. Rev. D 44, 3402 (1991); J. Felix et al. Phys. Rev. Lett. 82, 5213 (1999).

[2] L.G. Pondrom, Physics Report 122, 57 (1985); B. Anderson, G. Gustafson and G. Ingelman, Phys. Lett. B 85, 417 (1979); T.A. DeGrand and H.I. Miettinen, Phys. Rev. D 24, 2419 (1981); J. Soffer and N.A. Tőrnqvist, Phys. Rev. Lett. 68, 907 (1992); Y. Hama and T. Kodama, Phys. Rev. D 48, 3116 (1993); S.M. Troshin and N.E. Tyurin, Sov. J. Nucl. Phys. 38, 4 (1983); Phys. Rev. D 55, 1265 (1997); G. Domínguez Zacararías, G. Herrera, and I. León-Monzón, Eur. Phys. J. C 20, 677 (2001); G. Domínguez Zacararías and G. Herrera, Phys. Lett. B 484, 30 (2000).

[3] LiangZuo-tang, and MengTa-chung, Z. Phys. A 344, 171 (1992); Phys. Rev. D 49, 3759 (1994); C. Boros, and LiangZuo-tang, Phys. Rev. D 53, R2279 (1996); C. Boros, LiangZuo-tang, and MengTa-chung, Phys. Rev. Lett. 70, 1751 (1993); Phys. Rev. D 51, 4867 (1995); Phys. Rev. D 54, 4680 (1996).

[4] S. Saroff et al., Phys. Rev. Lett. 64, 995 (1990); D. Adams et al., Phys. Lett. B 261, 201 (1996); Z Phys. C 56, 181 (1992); A. Bravan et al., Phys. Lett. 75, 3073 (1995); D. Apokin et al., Phys. Lett. B 374, 319 (1990).

[5] As expected for the nature of the leading quarks in experimental results of asymmetries. See E791 Collaboration, E. M. Aitala et al., Phys. Lett. B 495, 42 (2000). 